\title{
Android and iPhone Mobile Apps for Psychosocial Wellness and Stress Management: Systematic Search in App Stores and Literature Review
}

Nancy Lau ${ }^{1,2}, \mathrm{PhD}$; Alison O'Daffer ${ }^{2}$, BA; Susannah Colt ${ }^{2}$, MA; Joyce P Yi-Frazier ${ }^{2}, \mathrm{PhD}$; Tonya M Palermo ${ }^{3,4}, \mathrm{PhD}$; Elizabeth McCauley ${ }^{1,4}, \mathrm{PhD}$; Abby R Rosenberg ${ }^{2,5,6}$, MD, MS, MA

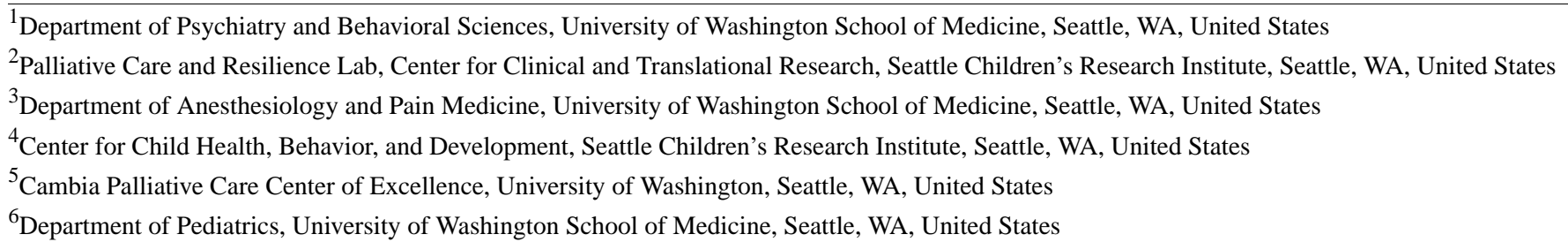

\section{Corresponding Author:}

Nancy Lau, PhD

Department of Psychiatry and Behavioral Sciences

University of Washington School of Medicine

1900 Ninth Ave

JMB 10-C

Seattle, WA, 98101

United States

Phone: 12068840569

Email: nancy.lau@seattlechildrens.org

\section{Abstract}

Background: In an oversaturated market of publicly available mobile apps for psychosocial self-care and stress management, health care providers, patients, and consumers interested in mental health-related apps may wonder which, if any, are efficacious. Readily available metrics for consumers include user popularity and media buzz rather than scientific evidence.

Objective: This systematic review aimed to (1) examine the breadth of therapeutic contents and features of psychosocial wellness and stress management apps available to self-help seekers for public download and (2) determine which of these apps have original research support.

Methods: First, we conducted a systematic review of publicly available apps on the iPhone App Store (Apple Inc) and Android Google Play (Google LLC) platforms using conventional self-help-seeking search terms related to wellness and stress. The results were limited to English-language apps available for free download. In total, 2 reviewers independently evaluated all apps and discussed the findings to reach $100 \%$ consensus regarding inclusion. Second, a literature review was conducted on the included apps to identify supporting studies with original data collection.

Results: We screened 3287 apps and found 1009 psychosocial wellness and stress management apps. Content varied widely. The most common evidence-based strategy was mindfulness-meditation, followed by positive psychology and goal setting. Most apps were intended to be used as self-help interventions, with only $1.09 \%$ (11/1009) involving an electronic therapist and $1.88 \%$ (19/1009) designed as a supplement to in-person psychotherapy. Only 4.66\% (47/1009) of apps targeted individuals with psychological disorders, and less than $1 \%$ of apps $(6 / 1009,0.59 \%)$ targeted individuals with other chronic illnesses. Approximately $2 \%(21 / 1009,2.08 \%)$ were supported by original research publications, with a total of 25 efficacy studies and 10 feasibility studies. The Headspace mindfulness app had the most evidence, including 8 efficacy studies. Most other scientifically backed apps were supported by a single feasibility or efficacy study.

Conclusions: Only 2.08\% (21/1009) of publicly available psychosocial wellness and stress management mobile apps discoverable to self-help seekers have published, peer-reviewed evidence of feasibility and/or efficacy. Clinicians and investigators may use these findings to help patients and families navigate the volume of emerging digital health interventions for stress management and wellness. 
(JMIR Mhealth Uhealth 2020;8(5):e17798) doi: 10.2196/17798

\section{KEYWORDS}

mHealth; mobile health; mental health

\section{Introduction}

\section{Background}

Within the past decade, smartphones have become ubiquitous in personal, social, and work life [1], irrespective of gender, race and ethnicity, and socioeconomic status [2]. Overall, 75\% of Americans own a smartphone, and $83 \%$ of them never leave home without it $[1,3]$. On average, a person checks his or her phone 150 times per day [4]. Owing to the pervasiveness of smartphones in modern day culture, technological innovations may be leveraged to disseminate in the moment behavioral change interventions designed to promote healthy behaviors [5]. There is a robust market for health apps, with 325,000 available for download as of 2017 and a growth rate of $25 \%$ year to year [6]. More than half of mobile phone users have downloaded a health-related mobile app, and the pace of development of evidence-based apps tested in research settings has lagged far behind than that of the commercial sector [7-9].

In particular, mobile health (mHealth) apps focused on promoting emotional health and adaptive coping have become increasingly popular. Mental health symptoms such as anxiety and stress are prevalent and disruptive. Overall, $75 \%$ of adults in the United States report significant stress, and 19\% have mental health disorders [10]. Anxiety disorders impact up to $30 \%$ of individuals worldwide, leading to severe societal and economic burden [11]. Work-related stress alone costs the US economy US \$402 billion [12]. Disseminating psychosocial interventions via mHealth technologies confers the advantage of universal accessibility regardless of geographic and economic restrictions $[1,13]$. According to the US National Comorbidity Survey (a nationally representative large-scale mental health study), common barriers to seeking mental health care include financial constraints, stigma, and a desire for self-management of symptoms [14]. In other studies, most individuals reported interest in using a mobile app for self-management of anxiety, stress, and depression if services were available for free $[8,15]$. In total, two recent meta-analyses of randomized controlled trials (RCTs) showed that mHealth interventions for anxiety and depression showed small positive effects when compared with an active control condition $[11,16]$.

Despite the high demand and potential advantages of these apps, there is a lack of quality control standards or readily accessible information to consumers on whether or which apps work in an oversaturated market. Thus, leveraging mHealth technologies brings both benefits and new challenges. In efforts to review publicly available apps using a direct-to-consumer approach, recent mHealth reviews have used a search strategy that involves entering key terms directly into the search engines of mobile app platforms [17-20]. In a review of iOS App Store mobile apps on Apple devices (Apple Inc), researchers identified 60 mobile apps that delivered at least one evidence-based stress management strategy (eg, mindfulness, progressive muscle relaxation, and biofeedback) [19]. A recent review of apps for depression, anxiety, posttraumatic stress disorder (PTSD), and alcohol use found that evidence-based mobile apps (ie, apps tested via formal research methods and published in the scientific literature) are often unavailable for download to the general public; in addition, apps available to consumers on commercial platforms are highly variable with regard to the inclusion of evidence-based content (ie, content derived from empirically supported therapeutic approaches) [20].

\section{Objectives}

With an overabundance of publicly available apps for stress management and psychosocial self-care, consumers may struggle with a paradox of choice, regardless of whether they are providers seeking to make app recommendations, patients seeking additional mental health support, or app-savvy digital natives interested in self-help. Readily available metrics are app visibility because of ranked lists, user popularity, media buzz, and user satisfaction ratings. When an app purports to be based in science, its scientific backing may not reach the classical standards of research rigor. It remains unclear whether popular apps that consumers gravitate toward work. In this study, we broadly reviewed popular apps available to all manner of consumers (ie, the general public, patients, and providers) for free download and their treatment content, user ratings, costs, and the evidence base in support of them. We presented findings from a review of 1009 publicly available mobile apps on Apple Store and Google Play (Google LLC) platforms using common self-help-seeking search terms for psychosocial wellness and stress management. Our review spanned Apple and Android devices that together represent $99 \%$ of the smartphone user market; $54.4 \%$ of US smartphone owners use Android devices and $44.3 \%$ use Apple devices [21]. After systematically searching both mobile app platforms (step 1), we supplemented this direct-to-consumer approach by conducting a literature review of the apps identified (step 2).

Our research questions were as follows: (1) What are the active therapeutic components and features of publicly available psychosocial wellness, coping, and stress management mobile apps? (2) Do any of these mobile apps have evidence in support of their feasibility/acceptability or efficacy in the published scientific literature? We hypothesized that the majority of consumer apps identified would not contain evidence-based therapeutic strategies, and even fewer would have published research supporting the apps themselves. We translated research findings to clinical practice by describing the breadth of popular wellness apps for stress management and by identifying the few apps with scientific backing.

\section{Methods}

\section{Searching the Apple Store and Google Play: Step 1}

We systematically identified and evaluated apps using a modified version of the Preferred Reporting Items for Systematic Reviews and Meta-Analyses guidelines [22]; adjustments were 
made because of the differing methodology of directly searching app store platforms. Our search strategy protocol is available in Multimedia Appendix 1.

We searched the mobile app platforms App Store iOS (Apple Inc) and Google Play in September 2018. Inclusion criteria were (1) a focus on stress management and/or psychosocial wellness, (2) available in English, and (3) free for download (including those with free downloads for basic subscriptions, with additional fees for extra features).

Specifically, we first created a list of conventional self-help-seeking search terms from mental health and positive psychology background literature [23-31]; we refined the list in discussions among our interdisciplinary research team, which includes intervention science researchers, health services researchers, physicians, social workers, and psychologists. Then, we entered into Apple Store and Google Play search engines the 14 conventional self-help-seeking search terms agreed upon by our team: stress, resilience, goal setting, relaxation, mindfulness, mood, coping, gratitude, optimism, hope, happiness, sadness, self-compassion, and self-care. We noted that the app results were displayed on both search engines in the order of popularity, based on proprietary algorithms. Hence, we screened the first 100 apps populated for each search term. Indeed, research suggests that smartphone users limit their searches to the first page of results (which contains 10 apps) [32], so screening the first 100 apps was deemed sufficient. In addition, we screened the popularity lists in health and fitness and kids and family categories for apps that met the inclusion criteria (these popularity lists are displayed on Apple and Google Play platforms). Two authors (NL and AO) independently reviewed all apps for inclusion and discussed the findings to reach $100 \%$ consensus. Duplicates were removed.

\section{Data Extraction Procedures}

For each app that met the inclusion criteria, 2 authors (NL and AO) extracted the following App Store iOS (Apple Inc) and Google Play product page data from November 2018 to February 2019 and discussed findings to reach $100 \%$ consensus: intervention and didactic content, target audience, and whether there were in-app paid features. In creating our database of intervention and didactic content, we used an all-inclusive approach to delineate content categories. For example, if an app description included meditation, mood tracking, artificial intelligence, and chat forums, we created unique content categories for each. Our goal was to provide a comprehensive representation of all intervention and didactic content as described on product pages by the app developers. To do so, we iteratively coded apps in sets of 50 and expanded the number of content categories as needed until there were no new content categories that arose. This resulted in the final version of the database, which contained 31 unique intervention and didactic content categories. During consensus conversations in March 2019 to April 2019, our process was to re-review App Store iOS (Apple Inc) and Google Play product page data to resolve discrepancies.

\section{Literature Review: Step 2}

After all mobile apps were identified in step 1, 2 authors (AO and SC) conducted a literature review via Google Scholar, MEDLINE, and PsycINFO databases using the search terms [app name] AND smartphone from April 2019 to June 2019 to identify peer-reviewed papers supporting each of the identified apps. Furthermore, 2 authors (NL and $\mathrm{AO}$ ) retrieved and independently reviewed the full text of all eligible studies to extract relevant feasibility and efficacy outcomes. We included research papers published in peer-reviewed journals and in English and included qualitative and/or quantitative studies with original data collection. We excluded conference presentations, editorials, commentaries, and study protocols. In consultation with a medical librarian, we chose to exclude nonpeer-reviewed scholarly works before publication in a peer-reviewed journal because the information included in conference abstracts lacks the rigor and external validity inherent in peer review. From the 33 included papers, we retrieved the following information: participants, sample size, study type, treatment conditions, and outcomes reported. Two authors (NL and AO) independently assessed study quality using the Cochrane collaboration's tool for assessing the risk of bias [33], evaluating random sequence generation (selection bias), allocation concealment (selection bias), blinding of participants and personnel (performance bias), blinding of outcome assessment (detection bias), incomplete outcome data (attrition bias), selective reporting (reporting bias), and other bias. We coded each category as low, high, or unclear risk of bias according to established standards. We resolved minor discrepancies in coding by referring to the journal papers themselves.

\section{Results}

\section{Searching the Apple Store and Google Play: Step 1}

We screened a total of 3287 apps (Figure 1). Of 3287 apps, 913 $(27.78 \%)$ were excluded after the initial screening process because they were not available for free download, not in English, or were duplicates. Of the remaining 2374 apps, 1251 $(52.70 \%)$ were excluded because they did not contain psychosocial wellness or stress management content (eg, health and fitness apps for exercise, nutrition, and weight loss). Of the remaining 1123 apps, $114(10.15 \%$ that we initially found on the Apple Store or Google Play no longer existed 3 months later when authors attempted to refer to the original source for consensus conversations in March 2019 to April 2019. We ultimately included 1009 apps in this review. 
Figure 1. Preferred reporting items for systematic reviews and meta-analyses diagram.
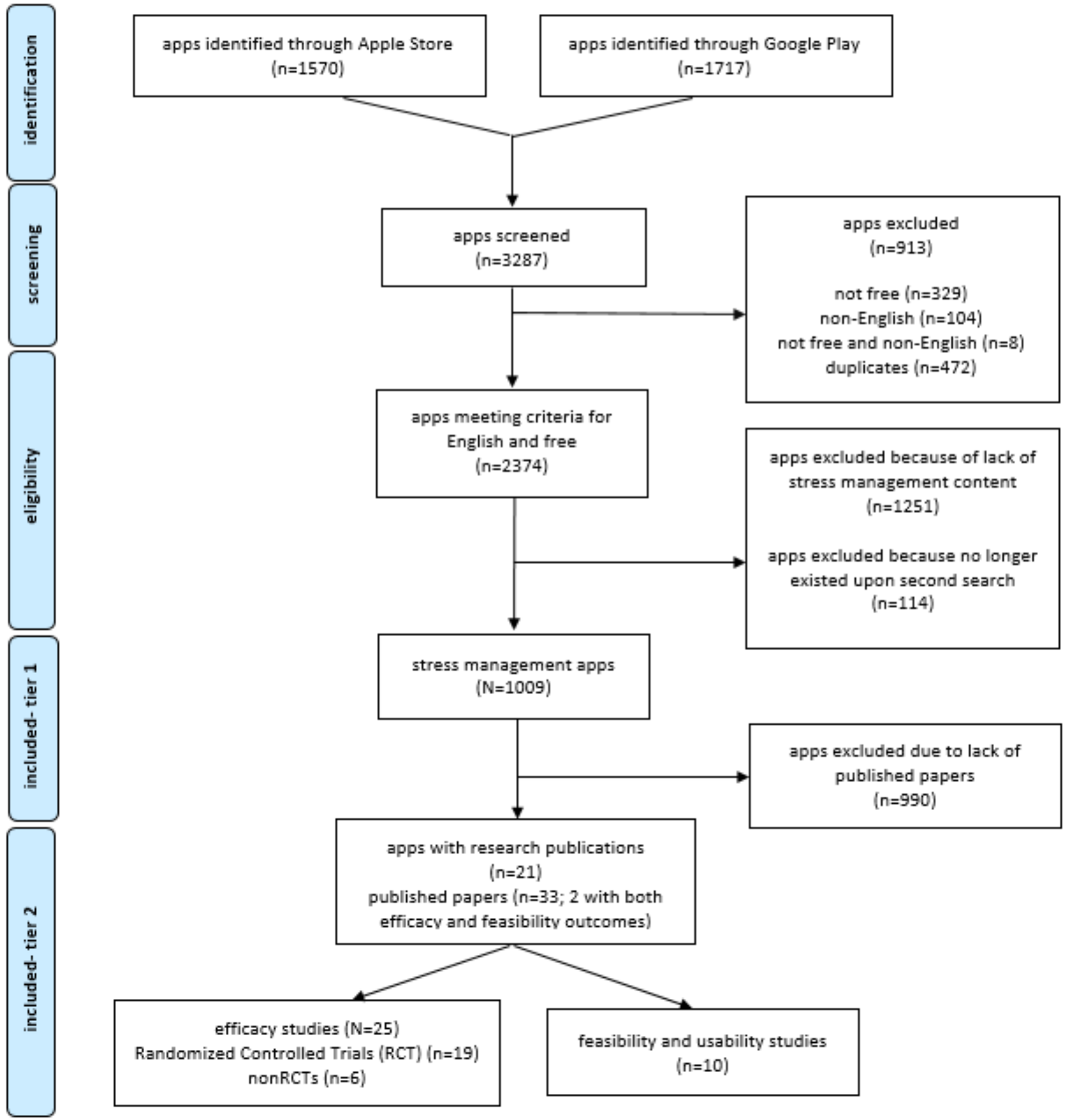

\section{Characteristics of All Included Apps (N=1009)}

For the pooled 1009 apps, we found 31 unique intervention and didactic content categories (eg, cognitive behavioral therapy, mindfulness-meditation, and journaling; Figure 2). Emotional-inspirational quotes were the most common app component, included in $22.99 \%(232 / 1009)$ of apps. Other common components included in $15 \%$ of apps or more were goal setting, positive psychology, journaling, music, mindfulness-meditation, and educational materials. Only $4.66 \%$ (47/1009) of apps were designed specifically for psychological disorders, less than $1 \%$ of apps were designed for chronic illnesses $(6 / 1009,0.59 \%)$, and $3.96 \%$ (40/1009) of apps were designed for youths and/or young adults. Most apps were intended to be used as self-help interventions, with only $1.09 \%$
(11/1009) involving an electronic therapist (e-therapist) and $1.88 \%$ (19/1009) designed as a supplement to in-person psychotherapy.

\section{Literature Review: Step 2}

\section{Characteristics of Subset of Apps With Research Support $(n=21)$}

We found supporting original research publications for $2.08 \%$ of apps (21/1009 identified). For this subset of apps, the most common therapeutic component was mindfulness-meditation, an evidence-based treatment strategy that was incorporated into $67 \%$ (14/21) of apps with published research followed closely by mood and symptom monitoring. All other common app features (included in $\geq 15 \%$ of apps) were also evidence-based 
treatment strategies: cognitive behavioral therapy, positive psychology, and relaxation (Multimedia Appendix 2 and Figure 3 ). For each app, the average user satisfaction ratings and the number of user ratings varied widely (Multimedia Appendix 3).

\section{Peer Review Publications}

A total of 33 peer-reviewed papers supported the 21 apps; 23 of these papers were efficacy studies [34-58], 8 were feasibility or usability studies, and 2 were combined efficacy and feasibility studies [39,59]. For each of the 21 apps with research support, the number of associated peer-reviewed publications ranged from 1 to 8 (Headspace [46,48-54]; Multimedia Appendix 3). The majority of apps $(16 / 21,76 \%)$ only had 1 publication [34,35,37,38,43,44,46,55,56,58-62]. 10\% Happier, Calm, and Headspace were the only research-supported apps we found on ranked health and fitness popularity lists.

Of the 25 efficacy studies, 19 were RCTs published between 2015 and 2019 [34-37,40-42,44-46,48,49,51-56,58,59], with 6 of the 19 trials testing Headspace [46,48-54]. The majority of studies used samples of convenience, that is, college students $[34,35,40,46,51,57]$ or users who had already downloaded the app [37,39,52,55,56,58]. Sample sizes for efficacy studies ranged from 19 [48] to 153,834 [39]. Treatment duration ranged from a single session of self-directed app use [39] to 6 months
[38]. All 16 apps with peer-reviewed publications that reported app efficacy showed some evidence of improving psychosocial outcomes over time (Multimedia Appendix 4). Studies collected varying outcome measures, ranging from unstructured self-directed app use $[41-45,50,62]$ to providing a sequential program of set frequency and duration $[49,53]$. In a subset of studies where effect sizes were reported; treatment effects ranged from small to large (Multimedia Appendix 4) [41,50,53,58]. Of the 10 apps with feasibility or usability studies, the majority (8/10 apps) reported that users found the apps to be enjoyable, accessible, and acceptable (Multimedia Appendix 5) [39,59-67]. Sample sizes for feasibility and usability studies ranged from 1 [60] to 1255 [64].

\section{Risk of Bias Assessment}

The risk of bias was evaluated for all 25 efficacy studies (Figure 4). Of the 19 RCTs, 18 reported random sequence generation and allocation concealment. For the blinding of participants and personnel domain and the outcome assessment domain, studies were roughly split in half between high and low risk; high-risk studies consisted of study designs with no control group, a waitlist control group, or an educational handout control group. For selective reporting bias, 6 were considered low risk, 1 high risk, and 18 were unclear. For other biases, 12 were considered low risk, 12 were high risk, and 1 was unclear.

Figure 2. Intervention and didactic content for all stress management apps $(\mathrm{N}=1009)$. Content categories were assigned based on descriptions by the app developer. Categories were not mutually exclusive, and a single app could be represented across one or more. E-therapist: electronic therapist.

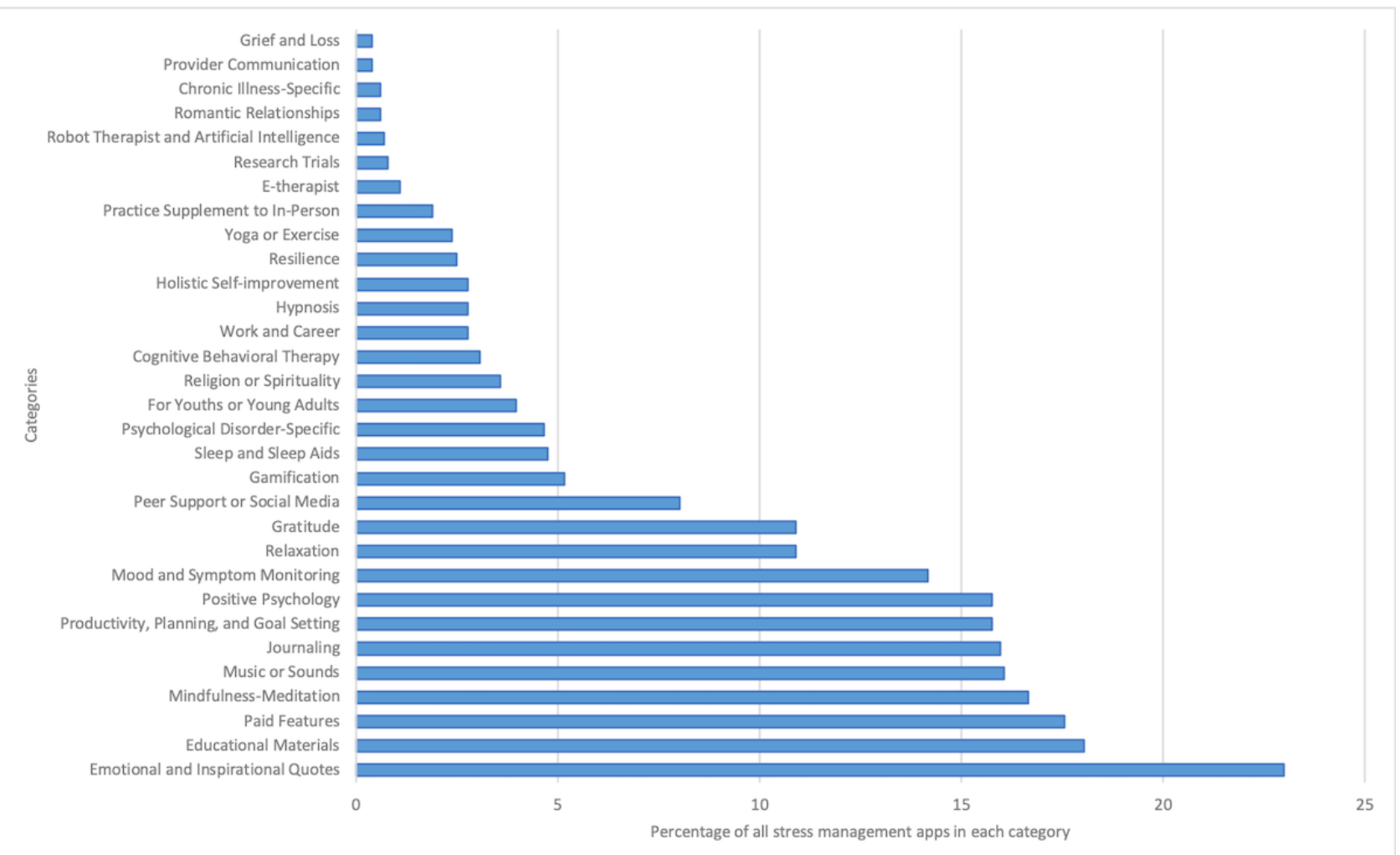


Figure 3. Intervention and didactic content for research-supported apps $(\mathrm{n}=21)$ vs all stress management apps (N=1009). Content categories were assigned based on descriptions by the app developer. Categories were not mutually exclusive, and a single app could be represented across one or more.

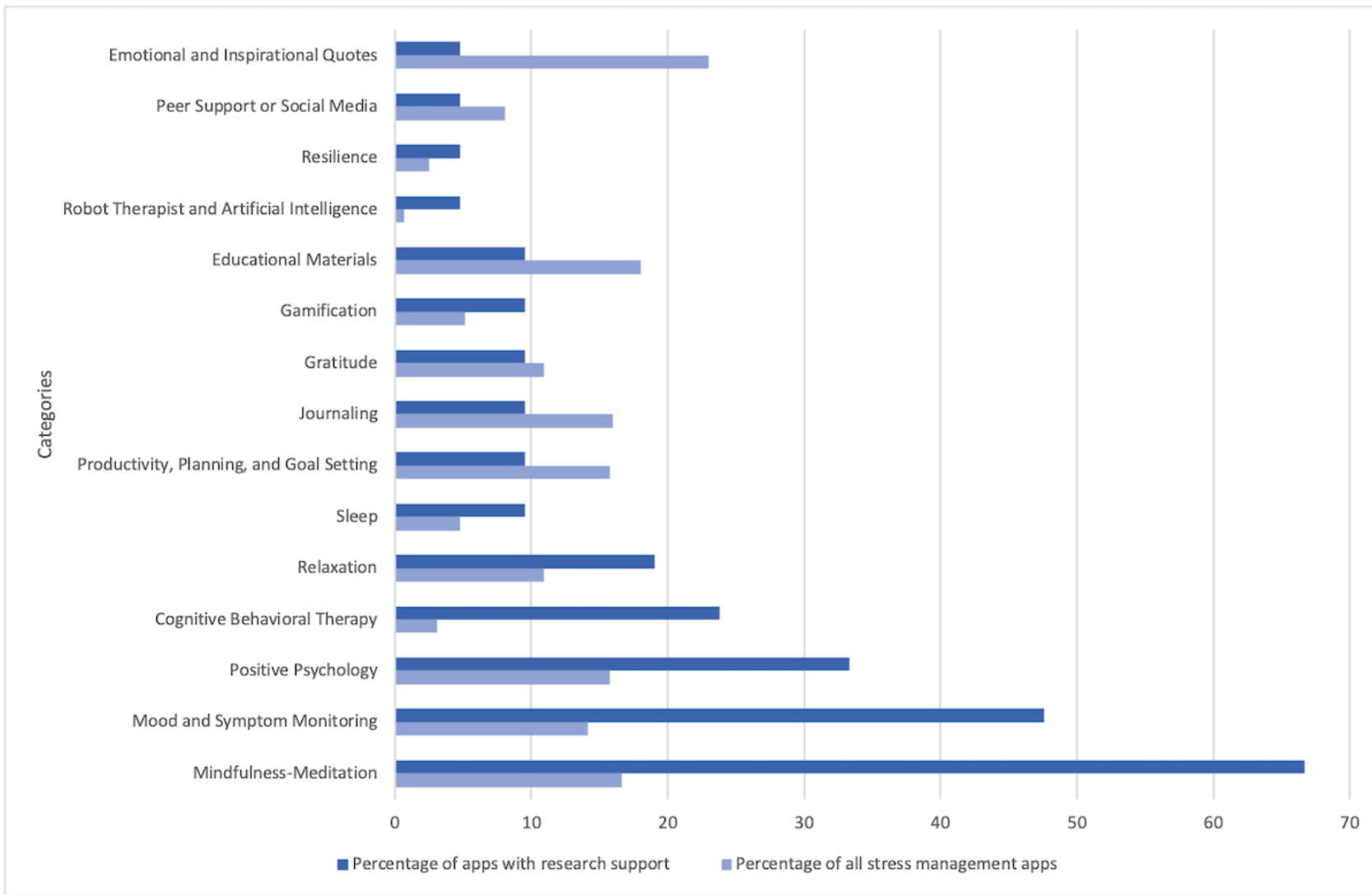


Figure 4. Summary of risk of bias.

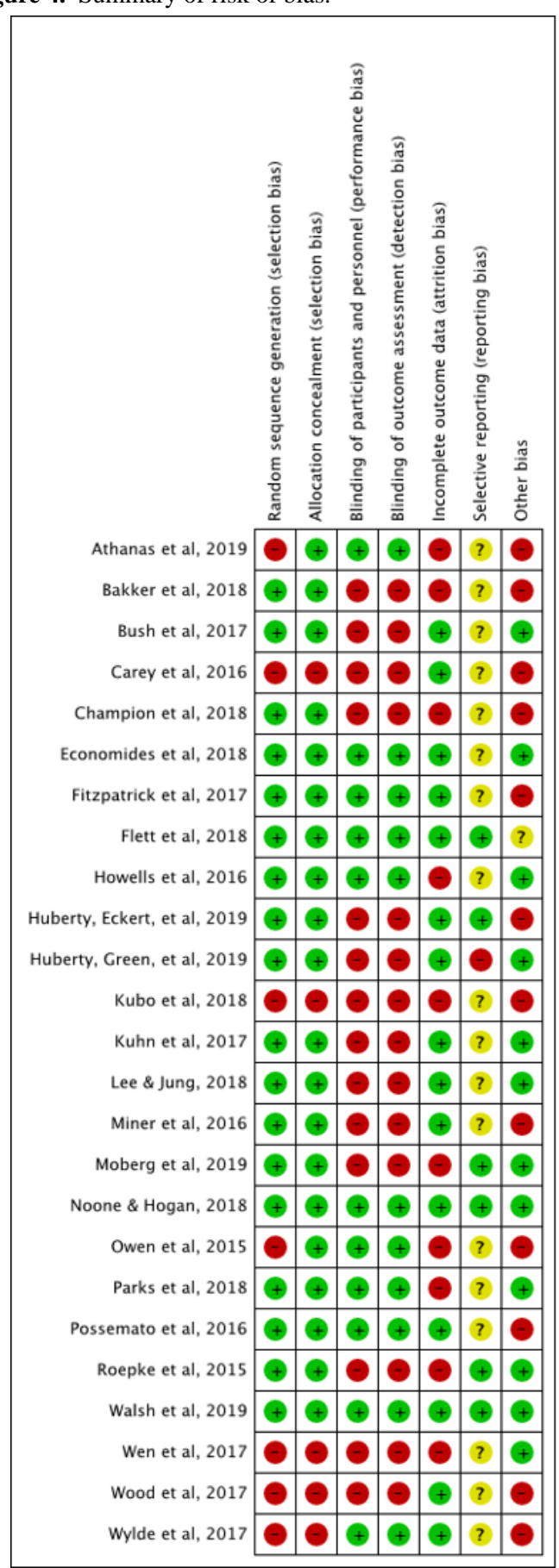

\section{Discussion}

\section{Principal Findings}

To our knowledge, this is the first review of psychosocial wellness and stress management apps using a multilevel search strategy of mobile app search engines (ie, what self-help consumers would find) followed by a literature review (ie, what scientists would find). We aimed to explore treatment features and components commonly folded into mainstream apps and whether and how these differed from those of apps tested in research and clinical trials. In addition, we summarized the existing literature on all identified apps.
We identified 1009 stress management and psychosocial wellness apps on the Apple Store and Google Play. App contents and features were varied and eclectic, ranging from journaling to hypnosis. Of the 5 most common treatment features and components for all apps, only 1 was evidence based (mindfulness-meditation). Unsurprisingly, the subset of apps with research publications was much more cohesive; the 5 most common therapeutic components identified in this group of apps were all evidence based. We found that 2.08\% (21/1009) of apps identified for inclusion had supporting research, and the majority of apps had only 1 research publication. All the published efficacy studies demonstrated some evidence that the app works, although effect sizes were rarely reported. However, the file-drawer problem [68] in academic research (ie, studies 
with null findings are less likely to be published) and contrary business incentives (ie, publishing null findings does not make for a marketable app) may contribute to potential publication biases that highlight positive effects. The majority of published feasibility studies demonstrated some evidence of user satisfaction and acceptability. Our research expands on findings from classic methodology systematic reviews of smartphone-based anxiety and depression interventions that have found small to moderate positive effects $[11,16]$.

Although there is a surfeit of mobile apps available for free download, only a small fraction of these have been tested in research settings. Even for the few published studies, the state of the science of mHealth for stress management and emotional wellness is in its nascent stages. Roughly half of the efficacy studies included in our review were either non-RCTs or RCTs without an active treatment comparison condition (ie, waitlist control). Approximately, one-fourth of all included studies were feasibility studies that did not measure efficacy outcomes. The majority of studies were not powered for analysis and, therefore, did not designate a priori primary vs secondary outcomes. Thus, the rapidly growing consumer market of mHealth for mental health is facing a similar research-practice gap to that of traditional face-to-face interventions: an overwhelming majority of self-help seekers may not be receiving evidence-based care [20]. This is not to discount the fact that businesses may have internally rigorous research and development processes outside of publications in scientific journals that we are unable to track or evaluate in a systematic way.

The World Health Organization (2019) recently released a guideline on digital health interventions for strengthening health systems based on an assessment of the benefits, harms, acceptability, feasibility, resource use, and equity considerations. The guideline's primary objective is the adoption of evidence-based interventions [69]. The European Commission provides complementary guidelines, including an assessment of data protection and privacy, safety, scientific content, and effectiveness [70]. In practice, mHealth interventions developed and tested in formal research settings for research purposes are rarely made available to the general public [71].

Potential future directions for traversing the research-practice divide are for academic researchers to partner with health technology companies and businesses to develop and test publicly available apps [72]. Such collaborations would improve the rigor of app development and continuous refinement by applying quality control standards to an unregulated market while capitalizing on the strengths of the commercial sector in financial and personnel resources, innovation, marketing, and motivational factors for user engagement. The app development process in the commercial sector adheres to a user-centered design framework, which engages end users as part of an iterative design process to better understand facilitators and barriers to sustainability and use [73]. This is a crucial model to apply in mHealth research because of historically low user adherence and retention rates. In addition, it is important to bolster the representation of other known evidence-based strategies in app components and features such as cognitive behavioral therapy, which was only represented in $3.07 \%$ (31/1009) apps identified. This was consistent with a previous review of popular anxiety and depression mobile apps that found evidence-based treatment strategies were poorly represented [74]. Finally, there is a need for comprehensive, consolidated, publicly available repositories of evidence-based stress management and psychosocial wellness apps going beyond consumer reports (including transparent information on public, private, or government ownership, public launch date, durability, and version history) so that the general public can make informed choices. Potential users should be directed to web-based resources such as PsyberGuide [75] to explore ratings and reviews for digital mental health products [71].

The majority of apps we identified were designed as self-help interventions; they were not necessarily intended for those with psychopathology. For individuals who are interested in seeking self-help via publicly available mHealth interventions, it is advisable to caution them against using this as a replacement to traditional treatment approaches, especially in the case of moderate to severe psychosocial problems. Importantly, the majority of studies found in our review used a sample of convenience (college students and users who have already downloaded the app and are therefore motivated to use them). It is unclear whether these apps would perform similarly if participants had more severe psychopathology symptoms.

Similarly, the role of health care providers and psychosocial clinicians in the mHealth space for an eclectic group of self-help seekers warrants exploration. E-therapist support is infrequently built into consumer apps $(11 / 1009,1.09 \%)$ of apps in our review). In addition to being resource intensive, this level of intervention may not be universally appealing or therapeutically indicated for generally healthy individuals interested in psychosocial self-care. On the other hand, in a clinical population with a serious mental health condition (PTSD), 1 of the studies included in our review found that clinician-supported app use outperformed self-directed app use [40]. This is consistent with previous literature regarding the therapist-patient relationship as a significant predictor of success in psychosocial treatment [76], and it remains to be determined whether app efficacy or engagement could be enhanced when paired with some form of clinician support. Future research should explore the optimal balance between clinician assistance and self-direction in mHealth and for which target audience (mental health support vs mental illness treatment support). At the very least, clinicians working with patients with diagnosed mental illnesses may choose to recommend specific evidence-based apps for skills practice and as a supplement to in-person therapy.

Recently, the Food and Drug Administration has released a timely policy report of its intent to provide regulatory oversight of mobile medical apps "for diagnosis of disease or other conditions, or the cure, mitigation, treatment, or prevention of a disease" and to "apply this oversight authority only to those software apps whose functionality could pose a risk to a patient's safety if the software apps were not to function as intended" [38]. Exceptions to oversight regulations are made for licensed professionals who create an app solely for use in their own practice or the manufacturing of mobile medical apps solely for use in research, teaching, and analysis and not for commercial use. This may influence the target audience for whom publicly available apps are developed as well as what is available for 
free. As the majority of apps identified in this review were advertised to consumers under a broad wellness and self-care umbrella, there are important gaps in addressing the unique psychosocial needs of vulnerable groups such as individuals with psychological disorders, individuals with chronic illnesses, and youths. Another important area of research is to explore how users engage with digital health technologies including the leveraging of big data analytics; identifying factors that can enhance engagement and usability will help inform the design and optimization of apps for long-term appeal and sustainability [5]. These factors have not yet been explored.

\section{Limitations}

There is no established gold standard for searching, evaluating, or reviewing digital health technologies. Recognizing that a methodology for using mobile app search engines to identify apps for review was nontraditional, we leveraged prior research on consumer apps to create a study-specific template and decision rules [17-19]. We relied on product pages on the Apple Store and Google Play to extract data on intervention characteristics, and specific search terms for our literature review which is not without its limitations. Although we provided transparency of our methods here, we recognized that they may not be reproducible. Similarly, it was impossible to construct a stable, final database of apps, given the quickly evolving mHealth landscape; new apps are developed, and old apps retired at a rapid rate. In the time frame in which our app store search was conducted, for example, $10.15 \%$ (114/1123) apps that were originally included in our database were no longer available 3 months later. Hence, our findings may lack the stability of classical systematic reviews. Similarly, it is possible that app names may have changed from the research design and testing phase reported in peer review publications to its official launch on the Apple Store or Google Play, or that other existing peer-reviewed publications may not have been identified utilizing our search strategy. For example, Happify's website lists two additional published efficacy studies that were not identified by our literature review search terms [77,78]. We were unable to conduct a meta-analysis of RCTs because of the differences in measures and measurement timepoints implemented across studies and relatively few trials with active comparison conditions.

Next, the apps we selected may not be representative of all mHealth programs. We included only free apps or free apps with in-app purchases. It is possible that paid apps significantly differ from free apps with respect to content and efficacy. Furthermore, some in-app purchases included access to an e-therapist, suggesting that secure services require greater monetary resources. As a result, access to effective digital health technologies may represent an unappreciated and important health disparity. However, it is unclear whether and the extent to which the involvement of an e-therapist bolsters outcomes and whether the benefits outweigh the costs; only $1.09 \%$ (11/1009) of apps included an e-therapist feature. In addition, the apps included in our review were limited to the English language. This may lead to a Western cultural bias in overrepresentation of apps with active coping strategies that reflect individualistic values and a personal sense of control over stressors [79-81].

\section{Conclusions}

In merging traditional systematic review methodologies with a direct-to-consumer selection criteria for mHealth apps, our study findings suggest that few publicly available stress management and psychosocial wellness apps that are discoverable to self-help seekers are evidence based. Additional research is needed regarding the relative role and utility of mHealth in individual self-care and the care of persons with serious mental illness, the value of mHealth-clinician collaborations, access to mHealth for patients with different resources, and the relative durability of mHealth impact. Meanwhile, clinicians, investigators, and consumers may use findings from our systematic review to navigate the volume of emerging digital health interventions for stress management and wellness.

\section{Acknowledgments}

NL is funded as an Implementation Science Scholar through the National Heart, Lung, and Blood Institute of the National Institutes of Health (grant number: 5K12 HL137940-02).

\section{Conflicts of Interest}

None declared.

\section{Multimedia Appendix 1}

Search strategies protocol. [DOCX File, 16 KB-Multimedia Appendix 1]

\section{Multimedia Appendix 2}

Table of intervention components of $n=21$ stress management apps with published research. [DOCX File, 26 KB-Multimedia Appendix 2]

\section{Multimedia Appendix 3}

Table of general information for $\mathrm{n}=21$ stress management apps with published research. 
[DOCX File, 22 KB-Multimedia Appendix 3]

\section{Multimedia Appendix 4}

Table of findings from efficacy studies $(n=25)$.

[DOCX File, $51 \mathrm{~KB}-$ Multimedia Appendix 4]

\section{Multimedia Appendix 5}

Table of usability or feasibility studies $(n=10)$.

[DOCX File, 26 KB-Multimedia Appendix 5]

\section{References}

1. Bakker D, Kazantzis N, Rickwood D, Rickard N. Mental health smartphone apps: review and evidence-based recommendations for future developments. JMIR Ment Health 2016 Mar 1;3(1):e7 [FREE Full text] [doi: 10.2196/mental.4984] [Medline: 26932350]

2. Anderson M, Jiang J. Pew Research Center. 2018. Teens, Social Media \& Technology URL: https://www.pewresearch.org/ internet/2018/05/31/teens-social-media-technology-2018/ [accessed 2020-04-02]

3. Pew Research Center. 2019. Mobile Fact Sheet URL: https://www.pewinternet.org/fact-sheet/mobile/, [accessed 2019-04-04]

4. Meeker M, Wu L. RMS Media: The Media Specialists. 2013. Internet Trends: D11 Conference URL: https://rmsmedia. co.za/wp-content/uploads/2013/08/Mary-Meeker-Internet-Trends-052913-final.pdf [accessed 2020-04-14]

5. Mohr DC, Burns MN, Schueller SM, Clarke G, Klinkman M. Behavioral intervention technologies: evidence review and recommendations for future research in mental health. Gen Hosp Psychiatry 2013;35(4):332-338 [FREE Full text] [doi: 10.1016/j.genhosppsych.2013.03.008] [Medline: 23664503]

6. Pohl M. Research2Guidance. 2017. 325,000 Mobile Health Apps Available in 2017-Android Now the Leading Mhealth Platform URL: https://research2guidance.com/ [accessed 2019-10-01]

7. Chib A, Lin SH. Theoretical advancements in mhealth: a systematic review of mobile apps. J Health Commun 2018;23(10-11):909-955. [doi: 10.1080/10810730.2018.1544676] [Medline: $\underline{\text { 30449261] }}$

8. Krebs P, Duncan DT. Health app use among us mobile phone owners: a national survey. JMIR Mhealth Uhealth 2015 Nov 4;3(4):e101 [FREE Full text] [doi: 10.2196/mhealth.4924] [Medline: 26537656]

9. Bose J, Hedden S, Lipari R, Park-Lee E. SAMHSA: Substance Abuse and Mental Health Services. 2017. Key Substance Use and Mental Health Indicators in the United States: Results from the 2018 National Survey on Drug Use and Health URL: https://www.samhsa.gov/data/sites/default/files/cbhsq-reports/NSDUHNationalFindingsReport2018/ NSDUHNationalFindingsReport2018.pdf [accessed 2019-08-01]

10. Anderson NB, Johnson SB, Belar C, Breckler S, Norda KC, Ballard D, et al. American Psychological Association. 2012. Stress in America: Our Health at Risk URL: https:/www.apa.org/news/press/releases/stress/2011/final-2011.pdf [accessed 2019-10-01]

11. Firth J, Torous J, Nicholas J, Carney R, Rosenbaum S, Sarris J. Can smartphone mental health interventions reduce symptoms of anxiety? A meta-analysis of randomized controlled trials. J Affect Disord 2017 Aug 15;218:15-22 [FREE Full text] [doi: 10.1016/j.jad.2017.04.046] [Medline: 28456072]

12. European Agency for Safety and Health at Work. 2014. Calculating the Cost of Work-related Stress And Psychosocial Risks URL: https://osha.europa.eu/en/publications/literature reviews/calculating-the-cost-of-work-related-stress-andpsychosocial-risks [accessed 2019-07-01]

13. Anderson M. Pew Research Center. 2019. Mobile Technology and Home Broadband URL: https://www.pewinternet.org/ 2019/06/13/mobile-technology-and-home-broadband-2019/, [accessed 2019-06-19]

14. Mojtabai R, Olfson M, Sampson NA, Jin R, Druss B, Wang PS, et al. Barriers to mental health treatment: results from the national comorbidity survey replication. Psychol Med 2011 Aug;41(8):1751-1761 [FREE Full text] [doi: 10.1017/S0033291710002291] [Medline: 21134315]

15. Proudfoot J, Parker G, Hadzi Pavlovic D, Manicavasagar V, Adler E, Whitton A. Community attitudes to the appropriation of mobile phones for monitoring and managing depression, anxiety, and stress. J Med Internet Res 2010 Dec 19;12(5):e64 [FREE Full text] [doi: 10.2196/jmir.1475] [Medline: 21169174]

16. Firth J, Torous J, Nicholas J, Carney R, Pratap A, Rosenbaum S, et al. The efficacy of smartphone-based mental health interventions for depressive symptoms: a meta-analysis of randomized controlled trials. World Psychiatry 2017 Oct;16(3):287-298 [FREE Full text] [doi: 10.1002/wps.20472] [Medline: 28941113]

17. Weekly T, Walker N, Beck J, Akers S, Weaver M. A review of apps for calming, relaxation, and mindfulness interventions for pediatric palliative care patients. Children (Basel) 2018 Jan 26;5(2):16 [FREE Full text] [doi: 10.3390/children5020016] [Medline: 29373515]

18. Greenle MM, Morgan B, Sayani S, Meghani SH. Identifying mobile apps targeting palliative care patients and family members. J Palliat Med 2018 Oct;21(10):1380-1385. [doi: 10.1089/jpm.2018.0157] [Medline: 30312126] 
19. Coulon SM, Monroe CM, West DS. A systematic, multi-domain review of mobile smartphone apps for evidence-based stress management. Am J Prev Med 2016 Jul;51(1):95-105. [doi: 10.1016/j.amepre.2016.01.026] [Medline: 26993534]

20. Livingston NA, Shingleton R, Heilman ME, Brief D. Self-help smartphone applications for alcohol use, PTSD, anxiety, and depression: addressing the new research-practice gap. J Technol Behav Sci 2019 May 9;4(2):139-151. [doi: 10.1007/s41347-019-00099-6]

21. Statista. 2020. Subscriber Share Held by Smartphone Operating Systems in the United States From 2012 to 2019 URL: https://www.statista.com/statistics/266572/market-share-held-by-smartphone-platforms-in-the-united-states/, [accessed 2019-07-01]

22. Moher D, Shamseer L, Clarke M, Ghersi D, Liberati A, Petticrew M, PRISMA-P Group. Preferred reporting items for systematic review and meta-analysis protocols (PRISMA-P) 2015 statement. Syst Rev 2015 Jan 1;4:1 [FREE Full text] [doi: 10.1186/2046-4053-4-1] [Medline: 25554246]

23. van Daele T, Hermans D, van Audenhove C, van den Bergh O. Stress reduction through psychoeducation: a meta- analytic review. Health Educ Behav 2012 Aug;39(4):474-485. [doi: 10.1177/1090198111419202] [Medline: 21986242]

24. Yates TM, Masten AS. Fostering the future: resilience theory and the practice of positive psychology. In: Linley PA, Joseph S, editors. Positive Psychology in Practice. New York City, USA: John Wiley \& Sons; 2004:521-539.

25. Farquharson L, MacLeod AK. A brief goal-setting and planning intervention to improve well-being for people with psychiatric disorders. Psychother Psychosom 2014;83(2):122-124. [doi: 10.1159/000356332] [Medline: 24457329]

26. Gu J, Strauss C, Bond R, Cavanagh K. Corrigendum to 'how do mindfulness-based cognitive therapy and mindfulness-based stress reduction improve mental health and wellbeing? A systematic review and meta-analysis of mediation studies' [clinical psychology review 37 (2015) 1-12]. Clin Psychol Rev 2016 Nov;49:119. [doi: 10.1016/j.cpr.2016.09.011] [Medline: 27776684]

27. Compas BE, Jaser SS, Bettis AH, Watson KH, Gruhn MA, Dunbar JP, et al. Coping, emotion regulation, and psychopathology in childhood and adolescence: a meta-analysis and narrative review. Psychol Bull 2017 Sep;143(9):939-991. [doi: 10.1037/bul0000110] [Medline: 28616996]

28. Davis DE, Choe E, Meyers J, Wade N, Varjas K, Gifford A, et al. Thankful for the little things: a meta-analysis of gratitude interventions. J Couns Psychol 2016 Jan;63(1):20-31. [doi: 10.1037/cou0000107] [Medline: 26575348]

29. Alarcon GM, Bowling NA, Khazon S. Great expectations: a meta-analytic examination of optimism and hope. Pers Individ Differ 2013 May;54(7):821-827. [doi: 10.1016/j.paid.2012.12.004]

30. Layous K, Lyubomirsky S. The how, why, what, when, and who of happiness: mechanisms underlying the success of positive activity interventions. Positive Emot 2014:473-495. [doi: 10.1093/acprof:oso/9780199926725.003.0025]

31. Anderson K, Burford O, Emmerton L. Mobile health apps to facilitate self-care: a qualitative study of user experiences. PLoS One 2016;11(5):e0156164 [FREE Full text] [doi: 10.1371/journal.pone.0156164] [Medline: 27214203]

32. Lieberman M. HubSpot Blog. 2014. 10 Stats About Inbound Marketing That Will Make Your Jaw Drop URL: https://blog. hubspot.com/insiders/inbound-marketing-stats [accessed 2019-11-01]

33. Higgins JP, Altman DG, Gøtzsche PC, Jüni P, Moher D, Oxman AD, Cochrane Bias Methods Group, Cochrane Statistical Methods Group. The cochrane collaboration's tool for assessing risk of bias in randomised trials. Br Med J 2011 Oct 18;343:d5928 [FREE Full text] [doi: 10.1136/bmj.d5928] [Medline: 22008217]

34. Fitzpatrick KK, Darcy A, Vierhile M. Delivering cognitive behavior therapy to young adults with symptoms of depression and anxiety using a fully automated conversational agent (Woebot): a randomized controlled trial. JMIR Ment Health 2017 Jun 6;4(2):e19 [FREE Full text] [doi: 10.2196/mental.7785] [Medline: 28588005]

35. Walsh KM, Saab BJ, Farb NA. Effects of a mindfulness meditation app on subjective well-being: active randomized controlled trial and experience sampling study. JMIR Ment Health 2019 Jan 8;6(1):e10844 [FREE Full text] [doi: 10.2196/10844] [Medline: 30622094]

36. Bush NE, Smolenski DJ, Denneson LM, Williams HB, Thomas EK, Dobscha SK. A virtual hope box: randomized controlled trial of a smartphone app for emotional regulation and coping with distress. Psychiatr Serv 2017 Apr 1;68(4):330-336. [doi: 10.1176/appi.ps.201600283] [Medline: 27842473]

37. Roepke AM, Jaffee SR, Riffle OM, McGonigal J, Broome R, Maxwell B. Randomized controlled trial of SuperBetter, a smartphone-based/internet-based self-help tool to reduce depressive symptoms. Games Health J 2015 Jun;4(3):235-246. [doi: 10.1089/g4h.2014.0046] [Medline: 26182069]

38. Athanas AJ, McCorrison JM, Smalley S, Price J, Grady J, Campistron J, et al. Association between improvement in baseline mood and long-term use of a mindfulness and meditation app: observational study. JMIR Ment Health 2019 May 8;6(5):e12617 [FREE Full text] [doi: 10.2196/12617] [Medline: 31066704]

39. Owen JE, Jaworski BK, Kuhn E, Makin-Byrd KN, Ramsey KM, Hoffman JE. mHealth in the wild: using novel data to examine the reach, use, and impact of PTSD coach. JMIR Ment Health 2015;2(1):e7 [FREE Full text] [doi: 10.2196/mental.3935] [Medline: 26543913]

40. Possemato K, Kuhn E, Johnson E, Hoffman JE, Owen JE, Kanuri N, et al. Using PTSD coach in primary care with and without clinician support: a pilot randomized controlled trial. Gen Hosp Psychiatry 2016;38:94-98. [doi:

10.1016/j.genhosppsych.2015.09.005] [Medline: 26589765] 
41. Miner A, Kuhn E, Hoffman JE, Owen JE, Ruzek JI, Taylor CB. Feasibility, acceptability, and potential efficacy of the PTSD Coach app: a pilot randomized controlled trial with community trauma survivors. Psychol Trauma 2016 May;8(3):384-392. [doi: 10.1037/tra0000092] [Medline: 27046668]

42. Kuhn E, Kanuri N, Hoffman JE, Garvert DW, Ruzek JI, Taylor CB. A randomized controlled trial of a smartphone app for posttraumatic stress disorder symptoms. J Consult Clin Psychol 2017 Mar;85(3):267-273. [doi: 10.1037/ccp0000163] [Medline: 28221061]

43. Wood AE, Prins A, Bush NE, Hsia JF, Bourn LE, Earley MD, et al. Reduction of burnout in mental health care providers using the provider resilience mobile application. Community Ment Health J 2017 May;53(4):452-459. [doi: 10.1007/s10597-016-0076-5] [Medline: 28070775]

44. Moberg C, Niles A, Beermann D. Guided self-help works: randomized waitlist controlled trial of Pacifica, a mobile app integrating cognitive behavioral therapy and mindfulness for stress, anxiety, and depression. J Med Internet Res 2019 Jun 8;21(6):e12556 [FREE Full text] [doi: 10.2196/12556] [Medline: 31199319]

45. Bakker D, Kazantzis N, Rickwood D, Rickard N. A randomized controlled trial of three smartphone apps for enhancing public mental health. Behav Res Ther 2018 Oct;109:75-83. [doi: 10.1016/j.brat.2018.08.003] [Medline: $\underline{\text { 30125790] }}$

46. Flett JA, Hayne H, Riordan BC, Thompson LM, Conner TS. Mobile mindfulness meditation: a randomised controlled trial of the effect of two popular apps on mental health. Mindfulness 2018 Oct 31;10(5):863-876. [doi:

$10.1007 / \mathrm{s} 12671-018-1050-9]$

47. Haug NA. PsyberGuide. 2016. Headspace: An Expert Review URL: https://www.psyberguide.org/expert-review/ headspace-expert-review/ [accessed 2019-07-01]

48. Kubo A, Altschuler A, Kurtovich E, Hendlish S, Laurent CA, Kolevska T, et al. A pilot mobile-based mindfulness intervention for cancer patients and their informal caregivers. Mindfulness (N Y) 2018 Dec;9(6):1885-1894 [FREE Full text] [doi: 10.1007/s12671-018-0931-2] [Medline: 30740187]

49. Wylde CM, Mahrer NE, Meyer RM, Gold JI. Mindfulness for novice pediatric nurses: smartphone application versus traditional intervention. J Pediatr Nurs 2017;36:205-212. [doi: 10.1016/j.pedn.2017.06.008] [Medline: 28888505]

50. Wen L, Sweeney TE, Welton L, Trockel M, Katznelson L. Encouraging mindfulness in medical house staff via smartphone app: a pilot study. Acad Psychiatry 2017 Oct;41(5):646-650. [doi: 10.1007/s40596-017-0768-3] [Medline: 28795335]

51. Noone C, Hogan MJ. A randomised active-controlled trial to examine the effects of an online mindfulness intervention on executive control, critical thinking and key thinking dispositions in a university student sample. BMC Psychol 2018 Apr 5;6(1):13 [FREE Full text] [doi: 10.1186/s40359-018-0226-3] [Medline: 29622047]

52. Howells A, Ivtzan I, Eiroa-Orosa FJ. Putting the 'app' in happiness: a randomised controlled trial of a smartphone-based mindfulness intervention to enhance wellbeing. J Happiness Stud 2014 Oct 29;17(1):163-185. [doi: 10.1007/s10902-014-9589-1]

53. Economides M, Martman J, Bell MJ, Sanderson B. Improvements in stress, affect, and irritability following brief use of a mindfulness-based smartphone app: a randomized controlled trial. Mindfulness (N Y) 2018;9(5):1584-1593 [FREE Full text] [doi: 10.1007/s12671-018-0905-4] [Medline: $\underline{\text { 30294390] }}$

54. Champion L, Economides M, Chandler C. The efficacy of a brief app-based mindfulness intervention on psychosocial outcomes in healthy adults: a pilot randomised controlled trial. PLoS One 2018;13(12):e0209482 [FREE Full text] [doi: 10.1371/journal.pone.0209482] [Medline: 30596696]

55. Parks AC, Williams AL, Tugade MM, Hokes KE, Honomichl RD, Zilca RD. Testing a scalable web and smartphone based intervention to improve depression, anxiety, and resilience: a randomized controlled trial. Int J Wellbeing 2018 Dec 8;8(2):22-67. [doi: 10.5502/ijw.v8i2.745]

56. Chittaro L, Vianello A. Evaluation of a mobile mindfulness app distributed through on-line stores: a 4-week study. Int J Hum-Comput St 2016 Feb;86:63-80. [doi: 10.1016/j.ijhcs.2015.09.004]

57. Huberty J, Green J, Glissmann C, Larkey L, Puzia M, Lee C. Efficacy of the mindfulness meditation mobile app 'calm' to reduce stress among college students: randomized controlled trial. JMIR Mhealth Uhealth 2019 Jun 25;7(6):e14273 [FREE Full text] [doi: 10.2196/14273] [Medline: 31237569]

58. Lee RA, Jung ME. Evaluation of an mhealth app (DeStressify) on university students' mental health: pilot trial. JMIR Ment Health 2018 Jan 23;5(1):e2 [FREE Full text] [doi: 10.2196/mental.8324] [Medline: 29362209]

59. Huberty J, Eckert R, Larkey L, Kurka J, de Jesús SA, Yoo W, et al. Smartphone-based meditation for myeloproliferative neoplasm patients: feasibility study to inform future trials. JMIR Form Res 2019 Apr 29;3(2):e12662 [FREE Full text] [doi: 10.2196/12662] [Medline: $\underline{31033443]}$

60. Diefenbach S, Müssig A. Counterproductive effects of gamification: an analysis on the example of the gamified task manager Habitica. Int J Hum Comput St 2019 Jul;127:190-210. [doi: 10.1016/j.ijhcs.2018.09.004]

61. Linette D, Bryant J. Managing Stress in Health Care With Meditation: Got a Minute? Am Nurse Today 2017;12(10):[FREE Full text]

62. Bush NE, Ouellette G, Kinn J. Utility of the T2 mood tracker mobile application among army warrior transition unit service members. Mil Med 2014 Dec;179(12):1453-1457. [doi: 10.7205/MILMED-D-14-00271] [Medline: 25469967] 
63. Carey TA, Haviland J, Tai SJ, Vanags T, Mansell W. MindSurf: a pilot study to assess the usability and acceptability of a smartphone app designed to promote contentment, wellbeing, and goal achievement. BMC Psychiatry 2016 Dec 12;16(1):442 [FREE Full text] [doi: 10.1186/s12888-016-1168-z] [Medline: $\underline{27955643]}$

64. Griauzde D, Kullgren JT, Liestenfeltz B, Ansari T, Johnson EH, Fedewa A, et al. A mobile phone-based program to promote healthy behaviors among adults with prediabetes who declined participation in free diabetes prevention programs: mixed-methods pilot randomized controlled trial. JMIR Mhealth Uhealth 2019 Jan 9;7(1):e11267 [FREE Full text] [doi: 10.2196/11267] [Medline: 30626566]

65. Bakker D, Kazantzis N, Rickwood D, Rickard N. Development and pilot evaluation of smartphone-delivered cognitive behavior therapy strategies for mood- and anxiety-related problems: MoodMission. Cogn Behav Prac 2018 Nov;25(4):496-514. [doi: 10.1016/j.cbpra.2018.07.002]

66. Kuhn E, Greene C, Hoffman J, Nguyen T, Wald L, Schmidt J, et al. Preliminary evaluation of PTSD coach, a smartphone app for post-traumatic stress symptoms. Mil Med 2014 Jan;179(1):12-18. [doi: 10.7205/MILMED-D-13-00271] [Medline: 24402979]

67. Bush NE, Dobscha SK, Crumpton R, Denneson LM, Hoffman JE, Crain A, et al. A Virtual Hope Box smartphone app as an accessory to therapy: proof-of-concept in a clinical sample of veterans. Suicide Life Threat Behav 2015 Feb;45(1):1-9. [doi: 10.1111/sltb.12103] [Medline: 24828126]

68. Rosenthal R. The file drawer problem and tolerance for null results. Psychol Bull 1979;86(3):638-641. [doi: 10.1037/0033-2909.86.3.638]

69. World Health Organization. WHO Guideline Recommendations On Digital Interventions For Health System Strengthening. Geneva, Switzerland: World Health Organization; 2019.

70. European Commission. 2017. Report of the Working Group on mHealth Assessment Guidelines URL: https://ec.europa.eu/ digital-single-market/en/news/report-working-group-mhealth-assessment-guidelines [accessed 2020-03-09]

71. Neary M, Schueller SM. State of the field of mental health apps. Cogn Behav Pract 2018 Nov;25(4):531-537. [doi: 10.1016/j.cbpra.2018.01.002]

72. Hingle M, Patrick H, Sacher PM, Sweet CC. The intersection of behavioral science and digital health: the case for academic-industry partnerships. Health Educ Behav 2019 Feb;46(1):5-9. [doi: 10.1177/1090198118788600] [Medline: $\underline{30041556]}$

73. Schnall R, Rojas M, Bakken S, Brown W, Carballo-Dieguez A, Carry M, et al. A user-centered model for designing consumer mobile health (mhealth) applications (apps). J Biomed Inform 2016 Apr;60:243-251 [FREE Full text] [doi: 10.1016/j.jbi.2016.02.002] [Medline: 26903153]

74. Wasil AR, Venturo-Conerly KE, Shingleton RM, Weisz JR. A review of popular smartphone apps for depression and anxiety: assessing the inclusion of evidence-based content. Behav Res Ther 2019 Dec;123:103498. [doi: 10.1016/j.brat.2019.103498] [Medline: 31707224]

75. PsyberGuide. URL: https://psyberguide.org/ [accessed 2019-03-09]

76. Martin DJ, Garske JP, Davis MK. Relation of the therapeutic alliance with outcome and other variables: a meta-analytic review. J Consult Clin Psychol 2000 Jun;68(3):438-450. [doi: 10.1037/0022-006x.68.3.438] [Medline: 10883561]

77. Williams A, Parks A, Cormier G, Stafford J, Whillans A. Improving resilience among employees high in depression, anxiety, and workplace distress. Int J Manag Res 2018 Dec;9(1-2):4-22.

78. Carpenter J, Crutchley P, Zilca RD, Schwartz HA, Smith LK, Cobb AM, et al. Correction: Seeing the "Big" Picture: Big Data Methods for Exploring Relationships Between Usage, Language, and Outcome in Internet Intervention Data. J Med Internet Res 2017 Dec 19;19(12):e347 [FREE Full text] [doi: 10.2196/jmir.8099] [Medline: 29258059]

79. Kuo BC. Culture's consequences on coping. J Cross Cul Psychol 2010 Sep 9;42(6):1084-1100. [doi: 10.1177/0022022110381126]

80. Chun C, Moos R, Cronkite R. Culture: a fundamental context for the stress and coping paradigm. In: Handbook of Multicultural Perspectives on Stress and Coping. Boston, MA: Springer; 2006:29-53.

81. O'Connor DB, Shimizu M. Sense of personal control, stress and coping style: a cross-cultural study. Stress Health 2002 Oct;18(4):173-183. [doi: 10.1002/smi.939]

\section{Abbreviations \\ e-therapist: electronic therapist \\ mHealth: mobile health \\ PTSD: posttraumatic stress disorder \\ RCT: randomized controlled trial}


Edited by G Eysenbach; submitted 14.01.20; peer-reviewed by Q Yuan, M Stuckey, A Tariq; comments to author 03.03.20; revised version received 17.03.20; accepted 22.03.20; published 22.05.20

Please cite as:

Lau N, O'Daffer A, Colt S, Yi-Frazier JP, Palermo TM, McCauley E, Rosenberg AR

Android and iPhone Mobile Apps for Psychosocial Wellness and Stress Management: Systematic Search in App Stores and Literature Review

JMIR Mhealth Uhealth 2020;8(5):e17798

URL: http://mhealth.jmir.org/2020/5/e17798/

doi: $\underline{10.2196 / 17798}$

PMID: $\underline{32357125}$

CNancy Lau, Alison O'Daffer, Susannah Colt, Joyce P Yi-Frazier, Tonya M Palermo, Elizabeth McCauley, Abby R Rosenberg. Originally published in JMIR mHealth and uHealth (http://mhealth.jmir.org), 22.05.2020. This is an open-access article distributed under the terms of the Creative Commons Attribution License (https://creativecommons.org/licenses/by/4.0/), which permits unrestricted use, distribution, and reproduction in any medium, provided the original work, first published in JMIR mHealth and uHealth, is properly cited. The complete bibliographic information, a link to the original publication on http://mhealth.jmir.org/, as well as this copyright and license information must be included. 